\title{
Australia, the forgotten continent, hitting above its weight in research
}

\author{
Michael R. Ditchfield
}

Received: 29 September 2014 / Accepted: 6 November 2014 / Published online: 2 December 2014

(C) Springer-Verlag Berlin Heidelberg 2014

Sir,

I read with great interest the article "Expert opinion: what are the greatest challenges and barriers to applying evidencebased and practical approaches to preclinical and clinical research in the field of pediatric radiology?" [1]. The article sought opinions from researchers in continents and subcontinents throughout the world. One continent was not included. Despite being the seventh-largest country by landmass, Australia is ranks 53rd in size by population [2] and therefore often forgotten in world opinion. Despite this, we are regular contributors to Pediatric Radiology. In the current edition, the review article of perianal disease in pediatric Crohn disease [3] and original article analysing MRI compliance in young children [4] are both Australian. This is not an isolated occurrence. From 2001 to 2010, Australia's health and medical research sector ranked sixth internationally in terms of citations per publication [5].

The valid challenges outlined in the article by Offiah et al. [1], including the rarity of many pediatric conditions and a need for more funding to enable more research time and improved technology, are relevant everywhere, including Australia. The article did not expand on the more important issue of overcoming these challenges, namely the clever use of limited resources and pursuit of opportunities when they arise. These include an interesting case series, a special interest resulting in a literature review or a PACS system that allows auditing of results and conditions. In medical imaging, we also need to look for opportunities to be a part of observational, case control or randomised control studies, which are usually performed in conjunction with our clinical colleagues. The article also did not include the importance of a research culture within a department, which instills a research interest in younger graduates and goes a long way to overcoming many other challenges.

I look forward to the sequel "Expert opinion: how we overcame the challenges and barriers ..." and hope that more continents will be included in this discussion.

Conflicts of interest None

\section{References}

1. Offiah CA, Andronikou S, Avni F et al (2014) Expert opinion: what are the greatest challenges and barriers to applying evidence-based and practical approaches to preclinical and clinical research in the field of pediatric radiology? Pediatr Radiol 44:1209-1212

2. List of countries by population. Wikipedia. http://en.wikipedia.org/ wiki/List_of_countries_by_population. Accessed 29 Sept 14

3. Compton GL, Bartlett M (2014) Perianal disease in pediatric Crohn disease: a review of MRI findings. Pediatr Radiol 44:1198-1208

4. Cahoon GD, Davison TE (2014) Prediction of compliance with MRI procedures among children of ages 3-12 years. Pediatr Radiol 44: 1302-1309

5. McKeon S (2012) Strategic Review of Health and Medical Research in Australia-Better Health Through Research. http://www. medalspercapita.com/\#medals-per-capita:2012. Accessed 29 Sept 14
M. R. Ditchfield $(\bowtie)$

Monash Health, Monash Medical Centre,

246 Clayton Rd., Clayton 3168, VIC, Australia

e-mail: michael.ditchfield@monashhealth.org 